\title{
Correlation among Growth Conditions, Morphology, and Optical Properties of Nanocolumnar InGaN/GaN Heterostructures Selectively Grown by Molecular Beam Epitaxy
}

\author{
Steven Albert, Ana Bengoechea-Encabo, Xiang Kong, Miguel A. Sánchez-Garcia, Achim Trampert, \\ and Enrique Calleja
}

\begin{abstract}
This work reports on the selective area growth mechanism of green-emitting InGaN/GaN nanocolumns with long InGaN sections $(330-410 \mathrm{~nm})$ grown by molecular beam epitaxy on $\mathrm{GaN} /$ sapphire. The evolution of the morphology of the InGaN segment is found to depend critically on the nominal III/V ratio as well as the diameter of the $\mathrm{GaN}$ section, both affecting the local $\mathrm{II/V}$ ratio at the top of the nanocolumn. Depending on the local III/V ratio, either
\end{abstract} metal or nitrogen rich, the diameter of the InGaN segment

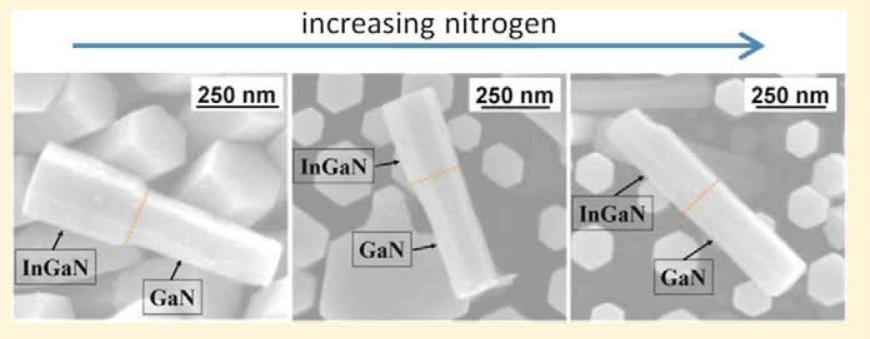
increases or decreases, respectively, compared to the initial diameter of the $\mathrm{GaN}$ segment. In addition, the In distribution inside the InGaN segment is found to depend on the local III/V and In/Ga ratios, i.e., increasing In content toward the top under local metal rich conditions and decreasing In content toward the top under locally $\mathrm{N}$ rich conditions.

\section{INTRODUCTION}

Selective area growth (SAG) of GaN nanocolumns (NCs) $)^{1,2}$ as well as InGaN/GaN NCs with thick InGaN segments ( 500 $\mathrm{nm}$ ) on $\mathrm{GaN} /$ sapphire as well as $\mathrm{Si}(111)$ emitting in the whole visible spectrum has been developed in the past several years as a way to overcome the limitations of self-assembled (SA) $\mathrm{NCs}^{3-5}$ which undergo a strong dispersion in morphological, optical, and electrical characteristics. ${ }^{6}$ The improvement in NC homogeneity, inherent to SAG, is expected to facilitate the processing of devices such as light emitting diodes (LEDs), thus increasing performance and reproducibility. 7,8

Commercial LEDs with InGaN multiquantum well (MQW) structures typically reach the maximal external quantum efficiency (EQE) at a rather low current injection, below 10 $\mathrm{A} / \mathrm{cm}^{2}$. At higher current injections, the so-called efficiency "droop" is observed, which has been attributed to a variety of mechanisms, such as carrier overflow, defects, or Auger recombination. ${ }^{9-11}$ The contribution of Auger recombination and carrier overflow to the droop effect is thought to be drastically reduced by increasing the active region volume in the device, to lower the carrier density. ${ }^{12,13}$ However, if thick In $\mathrm{GaN}$ active regions instead of MQWs are considered, a rather poor structural and optical quality of high-In content InGaN films would limit the applicability of that approach. Because NCs are practically free of extended defects, as compared to two-dimensional layers, InGaN/GaN NCs with long InGaN sections may provide an effective and elegant way to reduce droop effects while ensuring a high internal quantum efficiency (IQE), particularly for green emission where droop effects are stronger. ${ }^{14}$ First, LED structures using long ordered InGaN NCs ( 500 nm) emitting in the blue, green, and yellow spectral range have already been described. ${ }^{8}$ Furthermore, selectively grown high-quality InGaN/GaN NCs with a long InGaN segment would be very appealing for solar cell applications, as well. However, until now, no analysis of the growth mechanism of selectively grown thick InGaN segments has been performed, although this information is essential for future device optimization.

This work reports on a detailed study providing insight into the correlation among growth conditions, morphology, and optical properties of selectively grown $\mathrm{InGaN} / \mathrm{GaN} \mathrm{NCs}$ with thick InGaN sections emitting in the green range.

\section{EXPERIMENTAL SECTION}

Three samples were grown in a Riber Compact $21 \mathrm{MBE}$ system equipped with a rf-plasma source for active nitrogen and standard Knudsen cells for $\mathrm{Ga}$ and $\mathrm{In}$. The molecular fluxes were calibrated in (0001) $\mathrm{GaN}$ and InN growth rate units (nanometers per minute). ${ }^{15,16}$ All samples were grown using nanohole titanium masks fabricated by colloidal lithography on commercial (0001) GaN/sapphire templates (Lumilog). Details of the mask preparation can be found elsewhere. ${ }^{17}$

In all cases, nanoholes were arranged in a compact hexagonal lattice, with a pitch of $\sim 270 \mathrm{~nm}$ and an average diameter of $170 \mathrm{~nm}$. The initial $\mathrm{GaN}$ NC sections were grown on $\mathrm{GaN} /$ sapphire templates with a Ga flux $\left(\Phi_{\mathrm{Ga}}\right)$ of $18 \mathrm{~nm} / \mathrm{min}$ and a $\mathrm{N}$ flux $\left(\Phi_{\mathrm{N}}\right)$ of $5 \mathrm{~nm} / \mathrm{min}$, at 880 
${ }^{\circ} \mathrm{C}$ (thermocouple) for $3 \mathrm{~h}$ ( $\sim 450 \mathrm{~nm}$ long GaN sections). The InGaN sections were subsequently grown for $1 \mathrm{~h}$ on top of the GaN NCs under the conditions described in Table 1 . During InGaN growth, an

Table 1. Growth Conditions of the InGaN Sections and Low-Temperature PL Peak Positions of Grown Samples ${ }^{a}$

\begin{tabular}{|c|c|c|c|}
\hline sample & $\begin{array}{l}\text { impinging } \mathrm{N} \text { flux }(\mathrm{nm} / \mathrm{min}) \\
\left.\mathrm{s}^{-1} \mathrm{~cm}^{-2}\right)\end{array}\left(\times 10^{14}\right.$ atoms & $\begin{array}{l}\mathrm{II} / \mathrm{V} \\
\text { ratio }\end{array}$ & $\begin{array}{l}\text { PL peak } \\
(\mathrm{eV})\end{array}$ \\
\hline $\mathrm{Al}$ & $14(10.3)$ & 0.49 & 2.48 \\
\hline A2 & $17(12.5)$ & 0.40 & 2.36 \\
\hline $\mathrm{A} 3$ & $20(14.7)$ & 0.34 & 2.32 \\
\hline
\end{tabular}

${ }^{a} \mathrm{~A}$ constant $\mathrm{In} / \mathrm{Ga}$ ratio of 1.96 and a growth temperature of $650^{\circ} \mathrm{C}$ were used in all samples.

In/Ga ratio of 1.96 , with an In flux $\left(\Phi_{\mathrm{In}}\right)$ of $6.3 \mathrm{~nm} / \mathrm{min}\left(3.33 \times 10^{14}\right.$ atoms s $\left.\mathrm{s}^{-1} \mathrm{~cm}^{-2}\right)$ and a Ga flux $\left(\Phi_{\mathrm{Ga}}\right)$ of $2.3 \mathrm{~nm} / \mathrm{min}\left(1.7 \times 10^{14}\right.$ atoms $\mathrm{s}^{-1} \mathrm{~cm}^{-2}$ ), was used for all samples. After growth, the samples were characterized by scanning electron microscopy (SEM) using an FEI Inspect F50 instrument additionally equipped with a Gatan Mono CL4 unit allowing for cathodoluminescence (CL) measurements and lowtemperature photoluminescence (PL) spectroscopy using a $\mathrm{He}-\mathrm{Cd}$ laser $(325 \mathrm{~nm})$ with a power density of $\sim 1 \mathrm{~W} / \mathrm{cm}^{2}$. The $(\mathrm{In}, \mathrm{Ga}) \mathrm{N} /$ $\mathrm{GaN}$ interface and In distribution in NCs were investigated by highresolution transmission electron microcopy (HRTEM) and low-loss electron energy-loss spectroscopy (low-loss EELS) in a JEOL 3010 microscope equipped with a postcolumn Gatan Enfina parallel electron energy-loss spectrometer.

\section{RESULTS AND DISCUSSION}

Figure 1a shows cross-section and top-view SEM pictures of samples $\mathrm{A} 1-\mathrm{A} 3$, as well as pictures of the corresponding PL emission color.

In the three cases, ordered InGaN/GaN NCs with a flat top facet, emitting in the green spectral range, were obtained. The growth rates of the InGaN sections, as determined from SEM pictures in Figure $1 \mathrm{~b}-\mathrm{d}$ (representative NCs of each sample), are $5.5,6.0$, and $6.8 \mathrm{~nm} / \mathrm{min}$ for samples $\mathrm{A1}-\mathrm{A} 3$, respectively, indicating a trend of increasing growth rate with increasing nitrogen concentration. Cross-section SEM pictures in panels $b$ and $\mathrm{c}$ of Figure 1 show an increasing diameter toward the NC top starting at (or below) the nominal InGaN/GaN interface, i.e., $40 \%$ increase for sample $\mathrm{Al}$ and $20 \%$ for sample A2. This diameter increase is rather abrupt for sample $\mathrm{A} 1$ at the $\mathrm{InGaN} /$ $\mathrm{GaN}$ interface (Figure $1 \mathrm{~b}$ ) and then gradual up to the top, whereas sample A2 shows a gradual diameter increase starting below the nominal InGaN/GaN interface (Figure 1c). However, for sample A3 (Figure 1d), the diameter beyond the $\mathrm{GaN} / \mathrm{InGaN}$ interface is almost constant, but for the topmost NC region where a sudden and asymmetric diameter reduction $(20 \%)$ is observed. Notice that the diameter comparison in panels $b-d$ of Figure 1 is done selecting on purpose individual NCs with the same starting $\mathrm{GaN}$ diameter of $\sim 170 \mathrm{~nm}$.

Figure 2 shows results of the structural investigation performed by TEM on an array of NCs of samples A1 and A3. The bright field TEM image of sample A1 (Figure 2a) reveals the morphology of the NCs in cross section, demonstrating their high degree of uniformity and flat top. Furthermore, an abrupt increase in the column diameter at the InGaN/GaN interface is observed. During high-resolution TEM (HRTEM) (Figure 2b), the presence of stacking faults at the interface region that cross the entire column (no formation of partial dislocations) is observed. The presence of these

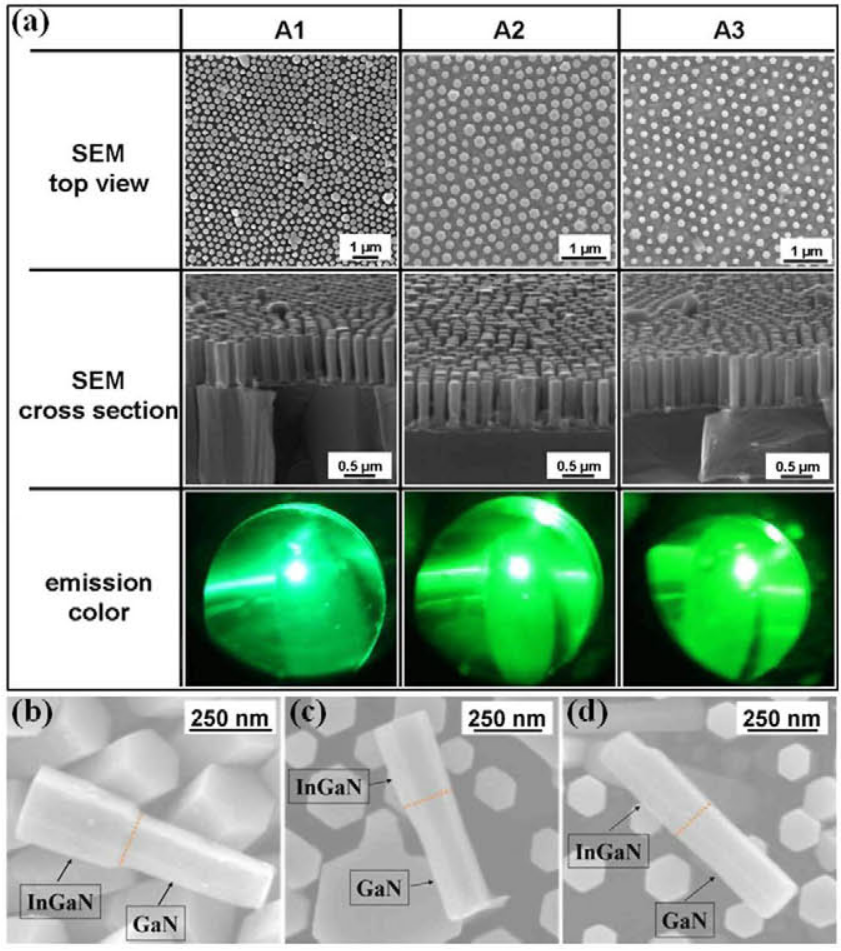

Figure 1. (a) SEM images and PL emission pictures of $\mathrm{InGaN} / \mathrm{GaN}$ NCs (A1-A3) and magnified cross-section SEM pictures of single $\mathrm{NCs}$ with a similar GaN section diameter $(\sim 170 \mathrm{~nm})$ of (b) sample $\mathrm{Al}$, (c) sample $\mathrm{A} 2$, and (d) sample $\mathrm{A} 3$, showing different diameter changes. The dotted lines indicate the position of the nominal InGaN/ $\mathrm{GaN}$ interface.
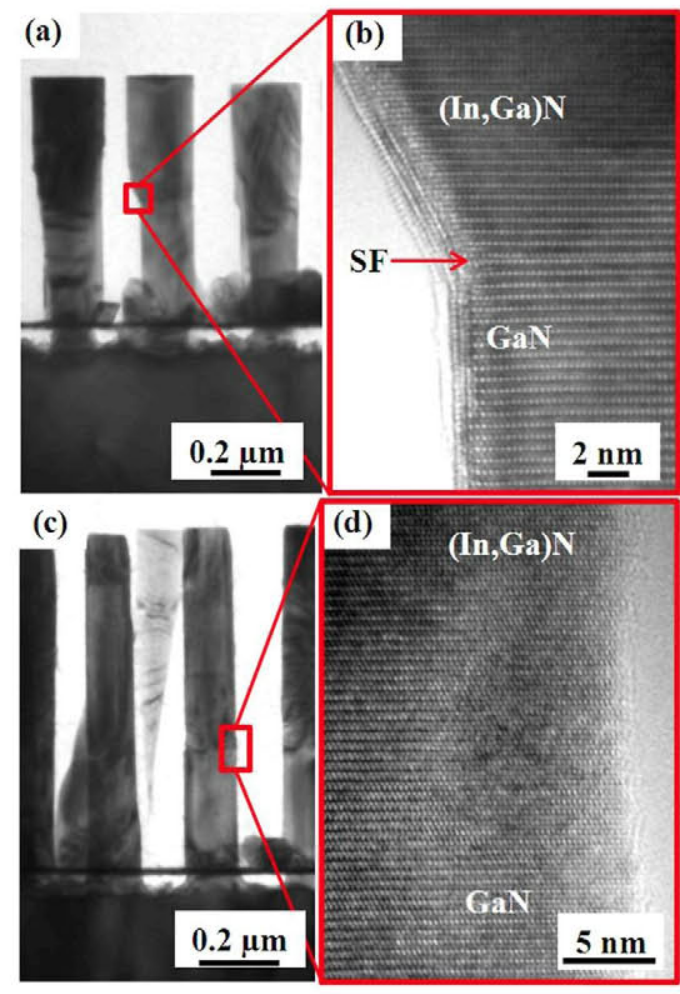

Figure 2. Bright field TEM picture of a NC array of (a) sample A1 and (c) sample A3. HRTEM pictures of the InGaN/GaN interface region in a single NC of (b) sample A1 and (d) sample A3. 
stacking faults (SFs) appears to be linked with the positions of the surface steps (diameter increase).

The bright field TEM image of sample A3 (Figure 2c) on the other hand reveals a uniform morphology across the InGaN/ $\mathrm{GaN}$ interface region, i.e., no abrupt change in NC diameter. Only a slight decrease in NC diameter toward the top is observed. The HRTEM images of both the InGaN/GaN interface region of sample $\mathrm{Al}$ (Figure $2 \mathrm{~b}$ ) and sample $\mathrm{A3}$ (Figure $2 \mathrm{~d}$ ) reveal a coherent interface between InGaN and GaN sections. In addition, selected area electron diffraction (SAED) patterns (not shown) taken at the interface region show no in-plane diffraction spot splitting. Both results together with the absence of misfit dislocations indicate that the lattice mismatch at the InGaN/GaN interface is accommodated elastically within the strained InGaN NCs. These results are in accordance with findings for SAG InGaN/GaN NCs grown on GaN-buffered $\mathrm{Si}(111)$. $^{18}$

Because we had taken advantage of the colloidal lithography process, during which a certain nanohole diameter variation is present, Figure 3 shows individual NCs from sample A3 with different starting $\mathrm{GaN}$ diameters. Here, the final shape and diameter of these NCs are found to depend strongly on the starting diameter.

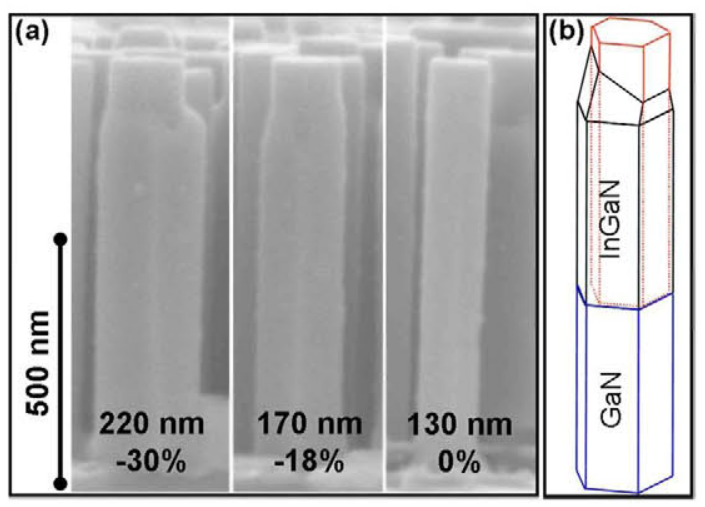

Figure 3. (a) Cross-section SEM pictures from InGaN/GaN NCs of sample $\mathrm{A} 3$ having different initial $\mathrm{GaN}$ diameters. The $\mathrm{GaN}$ diameters as well as the relative diameter change upon $\mathrm{InGaN}$ growth are shown. In all three cases, similar top diameters between 130 and $140 \mathrm{~nm}$ are observed. (b) Sketch showing the different contributions to the InGaN section growth in sample A3.

The different diameter changes observed in samples A1-A3 (or the absence of changes in sample A3 for a $130 \mathrm{~nm}$ starting diameter) seem to be closely related to the nominal III/V ratio and the initial GaN NC diameter. Both factors affect the "local" III/V ratio on the NC top, which depends on the directly impinging metal flux and the diffusion flux along the NC sidewalls toward its top.

It has been shown for SA GaN NCs that the NC diameter is directly related to the local $\mathrm{Ga} / \mathrm{N}$ ratio at the growth front (NC top) where the system always moves toward stoichiometry during growth. ${ }^{19} \mathrm{~A}$ similar mechanism is found to be present in the SAG InGaN/GaN NCs discussed here.

While no significant active nitrogen diffusion along the NC sidewalls occurs, ${ }^{20}$ the total amount of metal reaching the NC top depends strongly on the diffusion contribution up the NC sidewalls. Because of that, the total amount of metal atoms reaching the upper growth front (NC top) can be larger than the nominal impinging flux and is given by

$$
\Phi_{\text {metal }}^{\text {total }}=\Phi_{(\mathrm{In} / \mathrm{Ga})}^{\mathrm{imp}, \mathrm{top}}+\Phi_{(\mathrm{In} / \mathrm{Ga})}^{\mathrm{diff}}+\Phi_{(\mathrm{In} / \mathrm{Ga})}^{\mathrm{dec}}-\Phi_{(\mathrm{In} / \mathrm{Ga})}^{\mathrm{des}}
$$

All relevant contributions are shown in Figure 4.

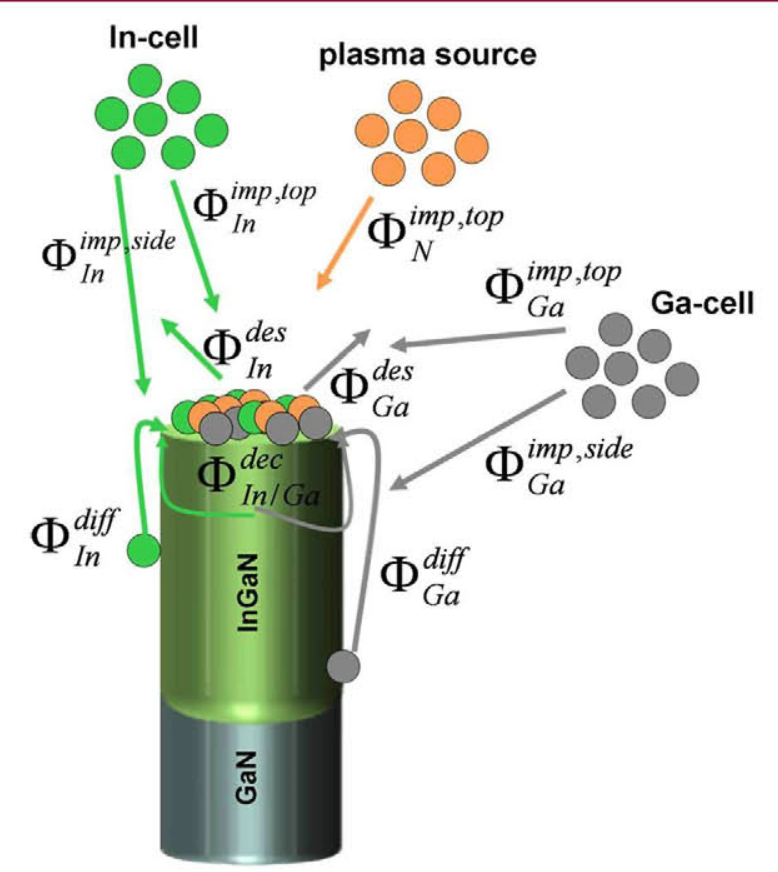

Figure 4. Sketch summarizing the contributions from different processes to the overall metal flux toward the NC top.

The decomposition fluxes ( $\Phi_{\mathrm{Ga}}^{\mathrm{dec}}$ and $\Phi_{\mathrm{In}}^{\mathrm{dec}}$ ) as well as desorption fluxes ( $\Phi_{\mathrm{Ga}}^{\text {des }}$ and $\Phi_{\mathrm{In}}^{\mathrm{des}}$ ) play a crucial role for the temperature used as can be seen by the rather small growth rates compared to the impinging fluxes. Given the fact that the same growth temperature was used in all three samples, decomposition and desorption are thought to be the same in all three samples. While the direct impinging metal fluxes $\left[\Phi_{\left(\mathbf{I n} / \mathrm{G}_{\mathrm{a}}\right)}^{\mathrm{im}, \text { top }}\right]$ are independent of the NC diameter, the metal diffusion flux $\left[\Phi_{(\mathrm{In} / \mathrm{Ga})}^{\text {diff }}\right]$ arriving to the NC top depends on the diameter of the NC as follows (from simple geometrical argumentation):

$$
\Phi_{(\mathrm{In} / \mathrm{Ga})}^{\mathrm{diff}} \propto \frac{\Phi_{\mathrm{In} / \mathrm{Ga}}^{\mathrm{imp}, \text { ide }} \times 2 \pi r L_{(\mathrm{In} / \mathrm{Ga})}^{\text {diff }}}{\pi r^{2}}=\Phi_{\mathrm{In} / \mathrm{Ga}}^{\text {imp,side }} \frac{2 L_{(\mathrm{In} / \mathrm{Ga})}^{\text {diff }}}{r}
$$

with $r$ being the NC radius and $L_{\mathrm{In} / \mathrm{Ga}}^{\mathrm{diff}}$ the diffusion lengths of $\mathrm{Ga}$ and In atoms along the c-direction at the NC sidewall. Then, an increase (decrease) in NC diameter leads to a net decrease (increase) in the diffusion flux toward the top. At this point, it has to be noted that the diffusion length of $\mathrm{Ga}$ atoms ( $\left.L_{\mathrm{Ga}}^{\text {diff }}\right)$ was found to be significantly greater than that of In atoms $\left(L_{\mathrm{In}}^{\text {diff }}\right)$ at the given growth temperature of $650{ }^{\circ} \mathrm{C}$, meaning that the diffusion flux contribution to the overall metal amount at the NCs top is higher for $\mathrm{Ga}^{21}$ For that reason, a diameter change would have more impact on the total $\mathrm{Ga}$ amount at the NC top. This will be a relevant point for the interpretation of the spatial indium distribution within the NC below.

The diameter increase observed in the InGaN section of samples $\mathrm{A} 1$ and $\mathrm{A} 2$ can be interpreted as originating from the local III/V ratio at the NC top being too high to reach stoichiometry for the given diameter defined by the $\mathrm{GaN}$ 

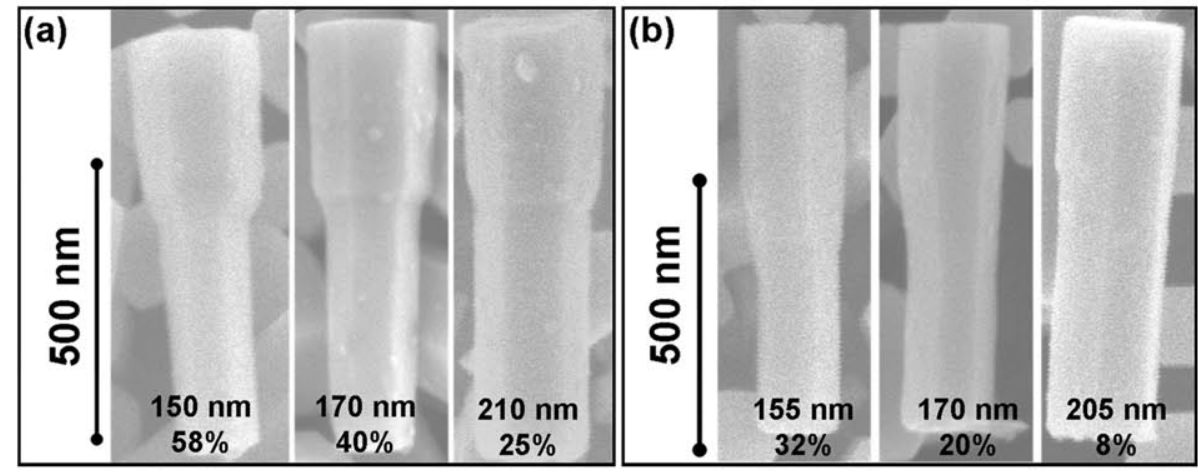

Figure 5. Diameter variation of InGaN sections in NCs of samples (a) A1 and (b) A2 starting from different initial GaN diameters (indicated). The diameter variations are indicated in percent.

section underneath (although ratios of nominal impinging fluxes are $<1$ ), that is

$$
\frac{\Phi_{\text {metal }}^{\text {total }}}{\Phi_{\mathrm{N}}^{\text {imp,top }}}>1
$$

at the beginning of the InGaN growth. To compensate for this metal excess at the NC top, its diameter increases to decrease the diffusion contribution of metal atoms toward the top. The greater the metal excess, the greater the diameter increase needed, as shown in panels $b$ and $c$ of Figure 1 for $I n G a N / G a N$ NCs with the same GaN diameter of $170 \mathrm{~nm}$ but different nominal III/V ratios (40\% in sample A1 grown with a nominal III/V ratio of 0.49 vs $20 \%$ in sample A2 grown with a nominal III/V ratio of 0.4). On the other hand, a net nitrogen excess at the growth front would lead to a diameter decrease, following the same mechanism to reach stoichiometry. In that case, the condition would be

$$
\frac{\Phi_{\text {metal }}^{\text {total }}}{\Phi_{\mathrm{N}}^{\text {imp,top }}}<1
$$

then causing a diameter decrease to increase the diffusion contribution of metal atoms.

In light of this model, the evolution of the InGaN section diameter should then depend on the fulfilled condition, defined by eq 3 or 4, until stoichiometry is reached. Figure 5 shows this dependence for NCs of samples $\mathrm{A} 1$ and $\mathrm{A} 2$ that start from different initial $\mathrm{GaN}$ diameters (because of diameter variations inherent to the colloidal lithography process).

It is clear from data in Figure 5 that the diameter increase of the InGaN section is determined by the condition imposed by eq 3 , that is, local metal excess at the NC top. In addition, it is clearly seen that a higher-metal excess condition (smaller GaN diameter) leads to a greater relative diameter change. The main behavior difference between samples $\mathrm{A} 1$ and $\mathrm{A} 2$ is the onset value of the initial diameter for the regime established by eq 3 , but not the trend that is similar. In sample A2, an initial diameter of $\sim 210 \mathrm{~nm}$ is nearly sufficient for reaching stoichiometry at the NC top (only a rather small diameter change of $\sim 8 \%$ of the InGaN section), whereas a similar diameter still leads to a significant diameter change in sample A1 upon InGaN growth (diameter change of $25 \%$ ). This difference is attributed to the different nominal impinging III/V ratios used (Table 1).

Considering the NCs from sample A3 shown in Figure 1d, with an initial GaN diameter of $170 \mathrm{~nm}$ (same as for samples $\mathrm{A} 1$ and $\mathrm{A} 2$ in panels $\mathrm{b}$ and $\mathrm{c}$, respectively, of Figure 1), the observed decrease in the InGaN top diameter (20\% smaller than the initial GaN one) suggests that the growth regime is determined by eq 4 , that is, local nitrogen excess. As one can see in Figure 3, an increase of the initial GaN diameter does not affect the InGaN top diameter; i.e., in both cases, the InGaN top diameter is $\sim 140 \mathrm{~nm}$. On the other hand, no change in NC diameter upon InGaN growth with respect to the $\mathrm{GaN}$ diameter is observed at all for a GaN starting diameter of $\sim 130$ $\mathrm{nm}$. A possible explanation is illustrated in the sketch shown in Figure $3 \mathrm{~b}$. The InGaN section grows under the regime given by eq 4 forcing a diameter reduction to a value determined by the $\mathrm{NC}$ top stoichiometry condition directly at the $\mathrm{InGaN} / \mathrm{GaN}$ interface. This will expose a portion of the initial $\mathrm{GaN} \mathrm{NC}$ top surface where growth can proceed as well but with a different growth rate. The combination of the two processes would give rise to a complex structure in which two different diameters are observed, inner and outer. This model would also predict that, for a sufficiently small initial $\mathrm{GaN}$ diameter, the growth front at the NC top would be at stoichiometry, so that no diameter changes would be expected. This is shown in Figure 3a for an initial diameter of $130 \mathrm{~nm}$.

PL spectra in Figure 6 show a clear red shift with an increasing level of nitrogen in samples $\mathrm{A} 1-\mathrm{A} 3$, pointing toward

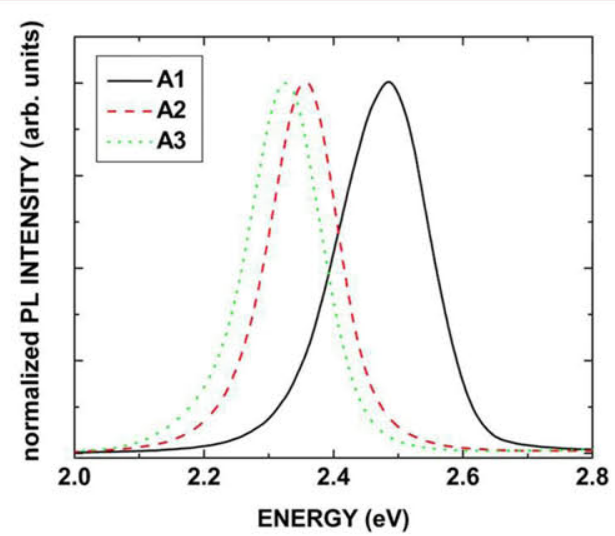

Figure 6. Low-temperature (7 K) PL spectra of samples A1-A3.

an increasing rate of In incorporation with an increasing level of nitrogen. However, this red shift, significantly pronounced between samples $\mathrm{A} 1$ and $\mathrm{A} 2$, is much smaller between samples $\mathrm{A} 2$ and $\mathrm{A} 3$. This behavior is expected, when moving from the metal rich to the nitrogen rich growth regime; ${ }^{22}$ i.e., samples $\mathrm{A} 1$ and $\mathrm{A} 2$ were grown under (local) metal rich growth conditions at the growth front (NC top), while in sample A3 (local), 


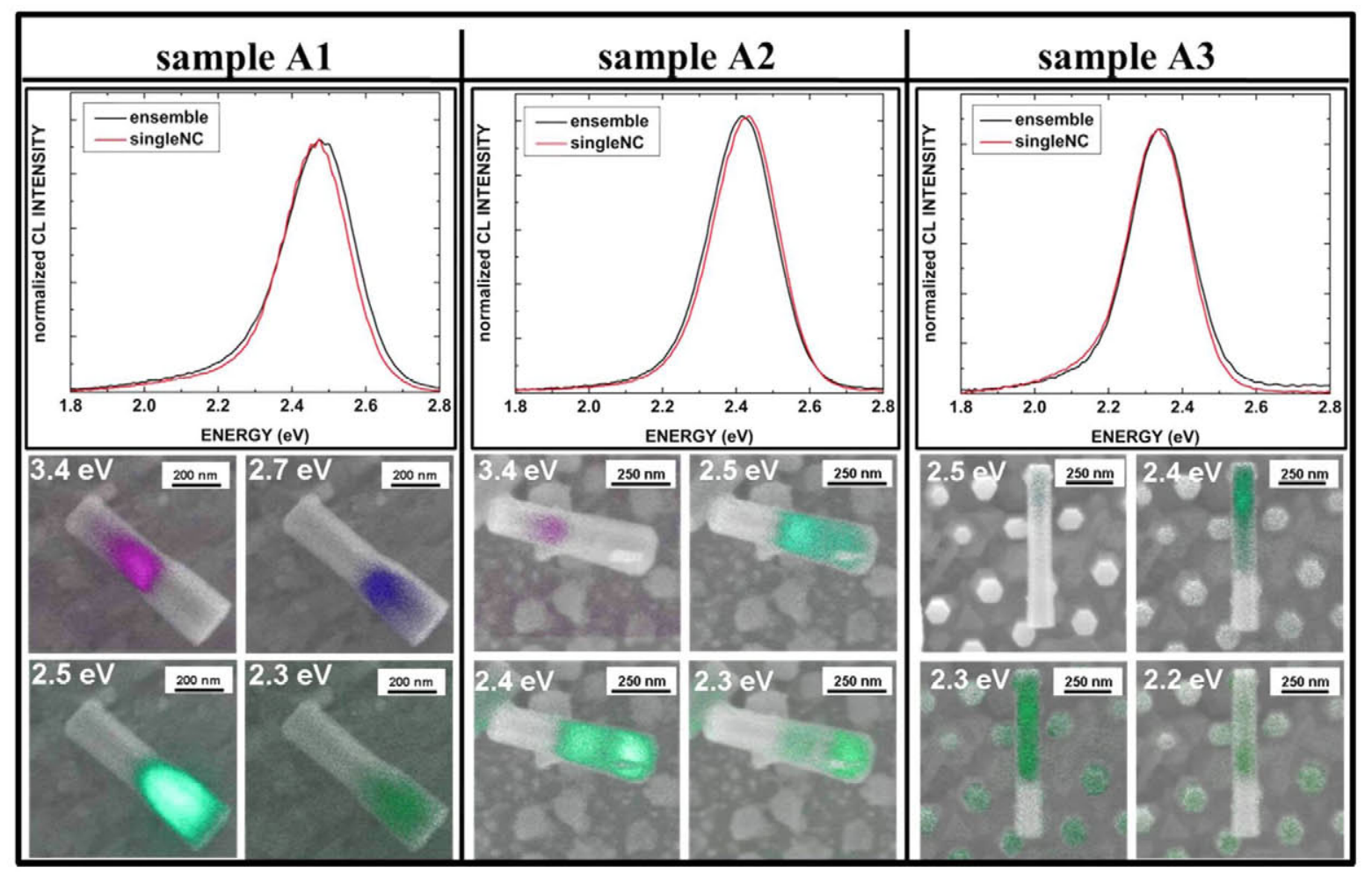

Figure 7. RT-CL spectra (top) of a single NC and an ensemble of $\sim 400 \mathrm{NCs}$ of samples A1-A3 measured. In addition, spatially resolved CL data from single NCs from samples $\mathrm{A} 1-\mathrm{A} 3$ are shown.

stoichiometric or $\mathrm{N}$ rich conditions were present, where all available In is incorporated. This finding supports the suggested model for the morphology evolution.

In addition to its impact on the NC morphology as well as the average In content determined by low-temperature PL, changes in the local III/V and In/Ga ratios at the NC top are expected to affect as well the axial In distribution inside the NCs. To gain information about the In distribution along the growth direction inside individual NCs, RT-CL measurements were performed on samples A1-A3 (Figure 7). Results show that spectra are identical for single NCs and ensembles, which points to a very high homogeneity and similar average level of In incorporation from NC to NC, as expected from SAG. As a consequence, the line width of $\sim 200 \mathrm{meV}$ in all three cases can be attributed solely to the specific In distribution within individual NCs.

Spatially resolved CL measurements of samples A1 and A2 (Figure 7) suggest the presence of a red shift of the emission toward the NC top, pointing toward an increase in the In composition along the NC, while the spatially resolved CL measurement of sample A3 indicates a blue shift of the emission toward the top, i.e., decreasing In content.

To obtain more information about the local In concentration and distribution inside NCs of samples $\mathrm{Al}$ and $\mathrm{A3}$, spatially resolved low-loss EELS was conducted along the axial direction in the center of the NCs using a spot size $\sim 10 \mathrm{~nm}$ in diameter. The bright field TEM images of the selected NCs, including the positions of the EELS measurements marked by small dots, are shown in Figure 8. The low-loss EELS scan of the single NC of sample A1 (Figure 8a) shows a general trend of increasing In content toward the top, whereas the low-loss EELS scan of a single NC of sample A3 (Figure 8b) reveals a decreasing In content toward the top. In addition, a reduced In content is observed at the InGaN/GaN interface region of both samples

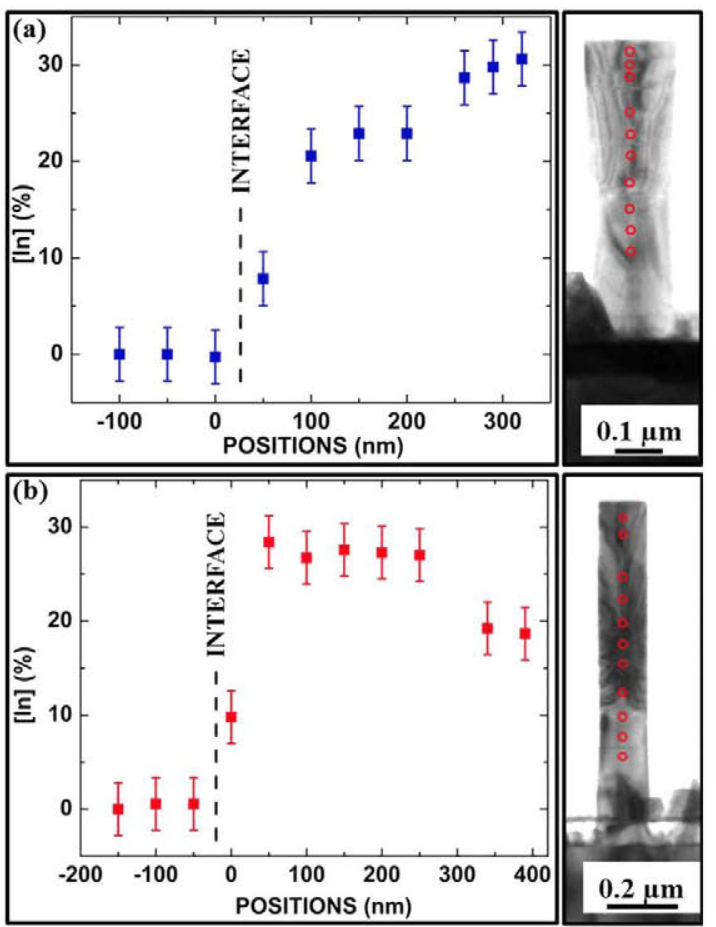

Figure 8. Indium concentration profiles measured by low-loss EELS and bright field TEM images of a single NC of (a) sample A1 and (b) sample A3. Circles in bright field TEM images indicate the points at which measurements were performed.

$\mathrm{Al}$ and $\mathrm{A} 3$, indicating the existence of a transition region, where the indium content gradually evolves and increases along the growth direction of the InGaN NCs. This result indicates the presence of a strain-driven lattice pulling effect in the InGaN/ 
GaN NC heterostructures, which is consistent with results observed before by Kong et al. ${ }^{18,23}$ At this point, it has to be noted that the width of this transition region is typically $\sim 50$ $\mathrm{nm}$ (in case of elastic relaxation). ${ }^{18}$

An increasing In content toward the top as observed for NCs of sample A1 was reported before for red-emitting SAG InGaN/GaN NCs grown on $\mathrm{GaN} /$ sapphire and attributed to partial InGaN decomposition and In segregation during the growth. ${ }^{3}$ A similar mechanism may be present here, as well. However, sample A3 shows a different behavior, i.e., a strong increase in In content close to the interface up to a "plateau value" of $\sim 28 \%$ (lattice pulling) followed by a decreasing In content toward the top. At this point, it has to be mentioned that all samples were grown at the same temperature $\left(650^{\circ} \mathrm{C}\right)$; hence, InGaN decomposition and In segregation can be thought to be similar in all cases. Because of that, an additional mechanism that gives rise to the observed changes in In content must be present. It is reasonable to assume that this mechanism relates to the evolution of morphology observed in samples A1-A3. With the increase in NC diameter (samples Al and A2), the amount (density) of metal atoms at the growth front (NC top) is reduced following eq 2, which determines the metal flux reaching the NC top by diffusion along the sidewalls. This reduction is the same for In and Ga fluxes along the sidewall, but given the differences between the directly impinging fluxes $\left(\Phi_{\mathrm{In}}=6.3 \mathrm{~nm} / \mathrm{min}\right.$ for In, and $\Phi_{\mathrm{Ga}}=2.3$ $\mathrm{nm} / \mathrm{min}$ for $\mathrm{Ga}$ ), the overall effect is a net increase in the In/Ga ratio at the growth front as the NC diameter increases (greater diffusion flux contribution for $\mathrm{Ga}$ than for In to total flux). In addition, the increasing diameter would lead to a reduction in the local $\mathrm{II} / \mathrm{V}$ ratio at the NC top, which leads as well to an increased level of In incorporation, given the local metal rich conditions at the NC top. ${ }^{22}$ This mechanism should not be present in an InGaN section with a constant or reduced diameter toward the NC top (sample A3), which is indeed observed (reduced In content toward the NC top) in Figure $8 \mathrm{~b}$. In principle, a diameter decrease leads to an increase in the local $\mathrm{Ga} / \mathrm{In}$ ratio, which in turn leads to a decreasing In content toward the top (blue shift) under local $\mathrm{N}$ rich conditions where all available In is incorporated. However, given the more complex morphology of InGaN segments of sample A3 for $\mathrm{GaN}$ diameters above $130 \mathrm{~nm}$ (abrupt diameter change at the NC top), no complete explanation for the observed blue shift can be provided at this point, and further studies will be needed to clarify this issue.

\section{CONCLUSION}

In summary, InGaN/GaN NCs with long InGaN segments $(330-410 \mathrm{~nm})$ emitting in the green spectral range were grown selectively on GaN/sapphire. The use of SAG allowed for a detailed and systematic study of the optical properties and growth mechanism of InGaN alloys, not possible before with self-assembled InGaN/GaN NCs because of their strong dispersion in the NC dimension and optical properties. The evolution of NC morphology as well as the distribution of In inside the InGaN/GaN NCs as a function of III/V ratio and NC diameter was explained by changes in the local III/V and In/Ga ratios at the NC top.

\section{AUTHOR INFORMATION}

Corresponding Author

*Edificio B, depacho B-039-1, Ciudad Universitaria s/n Madrid, 28040 Madrid, Spain. Phone: 0034915495700, ext. 4219. Fax: 0034914533567. E-mail: salbert@isom.upm.es.

\section{Author Contributions}

S.A. and A.B.-E. contributed equally to this work.

Notes

The authors declare no competing financial interest.

\section{ACKNOWLEDGMENTS}

We acknowledge partial financial support by EU FP7 Contract GECCO 280694-2 and by Spanish Projects CAM/P2009/ESP1503 and MICINN MAT2011-26703.

\section{REFERENCES}

(1) Sekiguchi, H.; Kishino, K; Kikuchi, A. Appl. Phys. Express 2008, $1,124002$.

(2) Bengoechea-Encabo, A.; Barbagini, F.; Fernandez-Garrido, S.; Grandal, J.; Ristic, J.; Sanchez-Garcia, M. A.; Calleja, E.; Jahn, U.; Luna, E.; Trampert, A. J. Cryst. Growth 2011, 325, 89-92.

(3) Albert, S.; Bengoechea-Encabo, A.; Sanchez-Garcia, M. A.; Calleja, E.; Jahn, U. J. Appl. Phys. 2013, 113, 114306.

(4) Albert, S.; Bengoechea-Encabo, A.; Kong, X; Sanchez-Garcia, M. A.; Calleja, E.; Trampert, A. Appl. Phys. Lett. 2013, 102, 181103.

(5) Albert, S.; Bengoechea-Encabo, A.; Sanchez-Garcia, M. A.; Kong, X.; Trampert, A.; Calleja, E. Nanotechnology 2013, 24, 1-8.

(6) Bavencove, A. L.j Tourbot, G.; Garcia, J.; Desieres, Y.; Gilet, P.; Levy, F.; Andre, B.; Gayral, B.; Daudin, B.; Dang, L. S. Nanotechnology 2011, 22, 1-9.

(7) Kishino, K.; Kamimura, J.; Kamiyama, K. Appl. Phys. Express 2012, 5, 031001.

(8) Bengoechea-Encabo, A.; Albert, S.; Lopez-Romero, D.; Lefebvre, P.; Barbagini, F.; Torres-Pardo, A.; Gonzalez-Calbet, J. M.; SánchezGarcia, M. A.; Calleja, E. Nanotechnology 2014, 25, 1-7.

(9) Piprek, J. Phys. Status Solidi A 2010, 207, 2217-2225.

(10) Kioupakis, E.; Rinke, P.; Delaney, K. T.; Van de Walle, C. Appl. Phys. Lett. 2011, 98, 161107.

(11) Vampola, K. J.; Iza, M.; Keller, S.; DenBaars, S. P.; Nakamura, S. Appl. Phys. Lett. 2009, 94, 061116.

(12) Gardner, N. F.; Müller, G. O.; Shen, Y. C.; Chen, G.; Watanabe, S.; Götz, W.; Krames, M. R. Appl. Phys. Lett. 2007, 91, 243506.

(13) Maier, M.; Köhler, K.; Kunzer, M.; Pletschen, W.; Wagner, J. Appl. Phys. Lett. 2009, 94, 041103.

(14) Kaneta, A.; Funato, M.; Kawakami, Y. Phys. Rev. B 2008, 78, 125317.

(15) Heying, B.; Averbeck, R.; Chen, L. F.; Haus, E.; Riechert, H.; Speck, J. S. J. Appl. Phys. 2000, 88, 1855-1860.

(16) Fernández-Garrido, S.; Grandal, J.; Calleja, E.; Sánchez-García, M. A.; López- Romero, D. J. Appl. Phys. 2009, 106, 126102.

(17) Bengoechea-Encabo, A.; Albert, S.; Sánchez-García, M. A; López, L. L.; Estradé, S.; Rebled, J. M.; Peiró, F.; Nataf, G.; de Mierry, P.; Zuniga-Perez, J.; Calleja, E. J. Cryst. Growth 2012, 353, 1-4.

(18) Kong, X.j Albert, S.; Bengoechea-Encabo, A.; Sánchez-García, M. A.; Calleja, E.; Trampert, A. Phys. Status Solidi A 2015, 1-4.

(19) Fernández-Garrido, S.; Kaganer, V. M.; Sabelfeld, K K; Gotschke, T.; Grandal, J.; Calleja, E.; Geelhaar, L.; Brandt, O. Nano Lett. 2013, 13, 3274-3280.

(20) Lymperakis, L.; Neugebauer, J. Phys. Rev. B 2009, 79, 241308(R).

(21) Sekiguchi, H.; Kishino, K.; Kikuchi, A. Appl. Phys. Lett. 2010, 96, 231104.

(22) Averbeck, R.; Riechert, H. Phys. Status Solidi A 1999, 176, 301305.

(23) Kong, X; Albert, S.; Bengoechea-Encabo, A; Sanchez-Garcia, M. A; Calleja, E.; Trampert, A. Nanotechnology 2012, 23, 1-6. 